BEHAVIOURAL CORRELATES OF ENERGY DRINK CONSUMPTION AMONG ADOLESCENTS: A REVIEW OF THE LITERATURE.

\title{
Authors:
}

Ade.Dawodu RN, BSc SCPHN, PgDip Adult Nursing Specialist Community Public Health Nurse (School Nurse) Child, Family, Health \& Wellbeing Directorate

Central London Community Healthcare NHS Trust (CLCH) Soho Centre for Health and Care London. W1D 3HZ.

Ade.Dawodu@clch.nhs.uk

Corresponding Author*

Dr Karen Cleaver * PhD, MSc, BEd (Hons) RGN/RSCN

Head of Family Care \& Mental Health

Faculty of Education \& Health

University of Greenwich

Avery Hill Campus

London SE9 2UG

k.p.cleaver@greenwich.ac.uk 020 8331-8075 


\title{
Behavioural Correlates of Energy Drink Consumption Among
}

\section{Adolescents: A Review of the Literature.}

\author{
Abstract: \\ Despite regulatory measures on the labelling of energy drinks, their \\ consumption among adolescents continues to grow in popularity, but \\ teachers increasingly report significant behavioural concerns among \\ students who disclose habitual energy drink consumption. A review of \\ papers published between January 2010 to October 2016 was \\ undertaken to determine whether an association between adverse \\ behaviour and consumption of energy drink exists. Findings indicate \\ that energy drink consumption among adolescents is associated with \\ alcohol and substance use, risky behaviours and psychological states \\ including: sensation-seeking, depression, and anxiety symptoms. \\ Energy drink consumption impacts negatively on executive functions, \\ and increases hyperactivity/inattention symptoms among adolescents. \\ An inverse association between energy drink consumption and sleep \\ duration exists. The findings highlight the need for greater awareness \\ of the potential risk of energy drink consumption among adolescents.

\section{Key Words} \\ Adolescents; Energy drinks; Risky Behaviours; Young People
}




\section{Introduction}

It is widely recognised that adolescents engage in risk taking behaviours, the causes of which are multiple including developmental, social, health and environmental influences (Leather, 2009). This paper explores concerns around a more recently identified risk taking behaviour, adolescent consumption of high levels of caffeine, through energy drinks. Energy drink (ED) consumption is growing in popularity amongst adolescents (Miyake and Marmorstein, 2015), with over two thirds of adolescents in the UK reported to consume them (ADEPIS, 2013). Growth in consumption is attributed to aggressive marketing strategies (Miller, 2008), their taste, the need for energy and combating sleepiness (ADEPIS, 2013). Increased consumption, ready availability, easy accessibility, and the limited regulation of EDs in the UK is increasingly a significant public health concern (ADEPIS, 2013, Visram \& Hashem, 2016).

\section{Background}

Energy drinks are caffeinated beverages that contain a mix of other energy promoting ingredients including; taurine, ginseng, guarana, and B-vitamins, and frequently sugar or sweeteners (Wolk et al, 2012). Caffeine is the main ingredient, ranging from 50 to $505 \mathrm{mg}$ per serving, three or more times the amount of caffeine present in soft drinks (Reissig et al, 2009, Seifert et al, 2011). Caffeine is known to provide the drink's energising effect through its ability to block 
adenosine from signalling the brain that the body needs to rest (Rockett and Putnam, 2002).

In adults, moderate consumption of energy drinks has been found to reduce perception of fatigue, improve alertness and concentration, enhance mental and physical performance, endurance, and reaction time (Ruxton, 2008). However, excessive caffeine intake has been associated with adverse health outcomes including cardiovascular symptoms, sleep impairment, nervousness, and gastrointestinal disturbances (Trapp et al, 2014), and death (Reissig et al, 2009). Adolescents are likely to be more susceptible to the adverse health risks of high caffeine content as they are not habitual caffeine users and have lower levels of tolerance for caffeine (Reissig et al, 2009).

Evidence, largely generated from the USA, indicates that many secondary school students consume EDs to increase energy levels and improve their sense of awareness, and have become habitual ED consumers, which can impact on their ability to focus and concentrate in school (Crane, 2009; Pennington et al, 2010). Teachers in the USA have reported more significant behavioural issues amongst students who have disclosed habitual consumption including tachycardia and dehydration requiring emergency intervention (Crane, 2009). The adverse effects of ED consumption has led to a doubling of emergency department attendances (Bonar et al, 2015), with 20,783 visits in 2011; $42 \%$ accounted for by $12-25$ year-olds (Substance Abuse and Mental Health Service Administration, 2013) 
Given the increasing popularity of ED consumption among adolescents, there is a surprising dearth of UK studies (Visram et al, 2105; Visram \& Hashem, 2016). Given the relative gap in research literature, this review aims to explore behavioural correlates of ED consumption amongst adolescents to determine lessons that might be applied for practise in the UK.

\section{Literature Search:}

A three-step strategy was undertaken to identify peer-reviewed papers published in English language between 2010 -2016. In stage one, EBSCOhost Databases which include: CINAHL; Medline; PsycINFO; Psychology and Behavioural Sciences Collection; Academic Search Premier; and SportDISCUS were searched. Search terms used were: “energy drink* AND (adolescen* OR teen* OR youth*) AND (risk* OR effect* OR behavio*)". The search was conducted by AA, the suitability of the papers for inclusion independently reviewed by both authors.

After the removal of duplicates, stage two entailed the screening of located papers for relevance. As the aim of this review was to explore the adverse behavioural correlates of energy drink consumption amongst adolescents, the titles and abstracts of the papers were assessed for eligibility using the following inclusion criteria: 
- Reported primary research;

- Reported behavioural outcomes on a human population not older than 18 years of age

- Reported outcomes on whole energy drink, not individual ingredients

- Reported on outcomes where energy drinks were the sole beverage i.e. not co-consumed with other beverages.

During stage three a Google Scholar search was conducted after handsearching the reference list of the eligible papers identified. A final search was conducted in October 2016.

The initial search yielded 601 papers. Applying the filters, peerreviewed papers published in English from 2010 to July 2015, and removing exact duplicates, limited the number to 228 papers. The abstracts of these papers were reviewed against the inclusion criteria resulting in the inclusion of twelve papers. Papers meeting the inclusion criteria were critically appraised and scored for methodological rigor using Critical Appraisal Skills Programme tools (CASP, 2013). This enabled the assessment of the methodology and reliability of the results of each of the eligible papers (Hannes et al, 2010). Each paper was graded as 'good', 'fair', 'poor', and 'very poor' based on their overall quality rating. Insert Figure 1 near here.

Twelve papers, all quantitative studies, mostly employing a cross sectional survey design were included. All papers employed self-report questionnaires largely administered on-line. It is recognised that social 
desirability, the tendency for individuals to present a favourable image of themselves, can confound survey/questionnaire results (Van de Mortel, 2008) undermining the validity of and reliability of research. Brener et al's. (2003) extensive review of the literature on adolescents' self-reports on a range of behaviours, including tobacco, alcohol, and other drug use, found that while self-reports are affected by cognitive and situational factors, this varied according to behaviour, as did the extent to which behaviours can be validated by objective measures (Brener et al, 2003). Nevertheless, Brener et al. (2003) note that it is important to assess adolescent risk taking behaviours and self-reports are the most effective way of capturing this data, researchers therefore need to be familiar with the inherent threats to validity these approaches entail.

Data was extracted from the eligible papers using a modified version of Hawker et al.'s. (2002) standardised table. (See Table 1). Insert Table 1 near here.

The primary outcome measures of the included papers were collated, organised and grouped, enabling the identification of recurrent findings, and the subsequent emergence of potential associations. An association with 'substance use' was drawn from a pattern, which emerged from 10 papers. Several measures associated with 'psychological wellbeing' were assessed; sensation seeking/risk taking was assessed as a primary outcome in 6 of the papers; other psychological outcomes including anxiety, depression, anger, and 
impulsivity and self-harming behaviour and suicidal thoughts were also examined. The influence of the adolescent-brain development on energy drink consumption was highlighted in the discussion section of 2 studies.

\section{Energy Drink and Alcohol/ Substance Use}

An association between energy drink consumption and alcohol and substance use was found in eight studies. Evren and Evren's. (2015) cross sectional study compared users and non-users of energy drinks among $10^{\text {th }}$ grade Turkish students; energy drink consumption was associated with heightened risk for lifetime tobacco, alcohol, and illicit drug use, with more frequent consumption having a stronger connection with substance use. Likewise, Terry-McElrath et al's. (2014) study, which examined the association between energy drink and soft drink consumption and substance use among American secondary school students, found a strong and positive association between energy drink consumption and past 30-day alcohol, cigarette, and illicit drug use was reported. This association was further reported to be significantly stronger than the association between regular or diet soft drinks and substance use.

Azagba et al. (2014) assessed whether more frequent use of energy drinks among 7th, 9th, 10th and 12th grade Canadian students was associated with poorer health and behavioural outcomes. They found that involvement in risky behaviours including tobacco, alcohol, 
marijuana, and other drug and substance use was higher among consumers of energy drinks relative to non-users. Similalry, Hamilton et al. (2013) found that energy drink consumption was reported to be greatly associated with binge drinking in the previous month, tobacco and cannabis smoking in the previous year, and non-medicinal prescription drug use in the previous year.

Larson et al.'s. (2014) study, which examined patterns and behavioural correlates of sport and energy drink consumption among adolescents found that a minimum weekly consumption of energy drinks was significantly associated with cigarette smoking, a finding also apparent in Gallimberti et al.’s. (2013) study of consumption amongst 11 -13 year olds.

Several studies reported an association between energy drink use and early onset alcohol use (Emond et al, 2014; Miyake and Marmorstein, 2014; Novak and Jasionowski, 2015). Miyake and Marmorstein. (2014) reported that parental monitoring (albeit as reported by adolescents participating in the study) is a contributory factor to the association between initial frequency of energy drink consumption and later frequency of alcohol use amongst; Miyake and Marmorstein. (2014) concluded that parents were more likely to monitor their children's food and beverage intake if they closely monitor their children's behaviour and whereabouts. 


\section{Energy Drink and Psychological Outcomes}

An association between energy drink consumption and psychological outcomes was evident. In Hamilton et al's. (2013) study $38.8 \%$ of adolescents who reported high sensation seeking had consumed energy drinks in the previous week, compared to $15.5 \%$ of those who did not. Likewise, heightened sensation seeking and elevated depressive symptoms were among the outcome measures identified to be significantly associated with an increased likelihood of energy drink consumption in Azagba et al.'s. (2014) cross-sectional study; sensation seeking was found to be associated with adolescents who consumed energy drinks, the association linked with level of consumption.

Similarly, Evren and Evren. (2015) found that psychological states (including sensation seeking, depression, anxiety symptoms, anger and impulsivity scores) and involvement in other self-destructive (selfharming and suicidal thoughts) and risky behaviours (tobacco, alcohol, and other drug use) were associated with an increased likelihood of energy drink consumption among high school students. TerryMcElrath et al. (2014) found an association between high sensationseeking adolescent groups to both energy drink consumption and substance use, but suggest that any observed association might weaken after controlling for sensation seeking. 


\section{Energy Drinks, Sleep and Functioning Patterns}

Sleep is a critical element in adolescent brain maturation (Dahl and Lewin, 2002); three papers assessed sleep as an outcome measure. Jackson et al. (2013) hypothesised that consumers of energy drinks would be more likely than those consuming caffeinated beverages only, such as tea, coffee and soft drinks, to report disordered sleep. “Trouble sleeping", "mind racing", "headaches", and "nausea" were among the physiological effects found to be more commonly reported by adolescents who consumed energy drinks, although "trouble sleeping" and "headaches" were also among physiological effects commonly reported by the caffeinated-only beverage users. The physiological adverse effects studied (including "trouble sleeping") were less compelling after adjusting for age, gender, and other substance use. Nevertheless, the findings substantiate earlier reports which suggest that adolescents with higher caffeine consumption were more likely to have trouble sleeping and to be tired in the morning, compared with those who had low caffeine consumption (Orbeta et al, 2006).

Larson et al. (2014) studied and compared the health behaviours associated with different frequencies of energy drink consumption. Data from their study suggested that after controlling for race/ethnicity, grade, and socio-economic status; boys and girls who are regular consumers of energy drinks had approximately 18 and 12 minutes less 
sleep respectively, when compared with peers who consumed EDs infrequently, findings reflected in studies examining consumption of caffeine only drinks (Calamaro et al, 2009; Lodato et al, 2013).

Van Batenburg-Eddes et al.'s. (2014) study assessed the associations of ED consumption with executive functions, found that young adolescents who regularly consume EDs display poorer performance in executive functions than those who do not. Consuming (on average) at least one energy drink/day was also associated with more problems in self-reported behaviour regulation and metacognition. Similarly, Scwhartz et al. (2014) studied patterns of sweetened beverage consumption, including EDs, to determine whether an association between consumption and hyperactivity/inattention existed. As in other studies reviewed (Gallimbert et al, 2013; Azagba, 2014; Emond et al, 2014; Evren \& Evren, 2015; Nowak \& Jasionowski, 2015; Schwarz et al 2015), ED consumption was higher in boys than girls. An association between hyperactivity/ inattention was observed; adjusting for age, race/ ethnicity, sex, school lunch eligibility, family structure, and sugary food consumption, risk of these behaviours increased by $14 \%$ for each additional sweetened beverage consumed, risk increased by $66 \%$ when energy drinks were consumed.

\section{Discussion}

Evidence from the literature reviewed indicates that an association between consumption of energy drinks and alcohol and substance use, 
risky behaviours, and alterations in sleep and executive performance exists.

A recurring theme across the studies was the association between consumption of energy drinks and alcohol use. However, it is not clear whether drinking alcohol increases likelihood for energy drink consumption or vice versa. The link between energy drink use and alcohol assumption is important as research that has examined the concomitant use of energy drinks and alcohol has found this to be associated with a range of risky behaviours including increased risk of marijuana, cocaine, and ecstasy use (Mann et al, 2016; Snipes and Benotsch, 2013), alcohol-related aggressive behaviours, risky sexual behaviours, and increased likelihood of alcohol-related accidents and injuries (Azagba et al, 2013; Howland and Rohsenow, 2013; Howland et al, 2011; Marczinski et al, 2011). Moreover, as energy drinks counter the depressant effects of alcohol, thereby increasing alertness, a risk arises that individuals' perception of alcohol intoxication is reduced, leading to a longer timeframe of drinking, culminating in a higher risk of binge drinking (Arria et al, 2014; Patrick et al, 2014), further exacerbating the likelihood to exposure of the risks listed above. Evidence indicates that adult risk behaviours, particularly smoking and alcohol use when initiated in adolescence, is related to dependency in adulthood (Hale \& Viner 2012), thus raising awareness of these risk factors could prove to be an important preventative/early intervention measure. 
Findings from the review also suggest an association between energy drink consumption and psychological states (including sensation seeking, depression, anxiety symptoms, anger and impulsivity scores) and involvement in other self-destructive (self-harming and suicidal thoughts) and risky behaviours (tobacco, and substance misuse). A clear dose-response pattern was also reported. Energy drink consumption was found to impact negatively on executive functions, and increase hyperactivity/inattention symptoms among adolescents.

Despite findings of insignificant associations between energy drink consumption and sleep duration, an inverse association was argued to be more likely based on evidence reported on caffeine (the primary ingredient in energy drinks), which suggest that caffeine is strongly associated with lower sleep duration among adolescents. Sleep is a critical element in adolescent brain maturation (Dahl and Lewin, 2002), but caffeine consumption alters normal brain development during critical development periods (Temple, 2009). The need to educate and raise awareness of the detrimental effects of energy drink consumption on sleep in adolescents who consume energy drinks to counteract insufficient sleep, cannot be overemphasised (Carrier et al, 2009). More awareness of the wide range of behavioural deficits linked to sleep deprivation, such as attention problems, irritability, behaviour regulation problems, and reduced metacognitive skills (Beebe et al., 2008; O’Brien, 2009) is also necessary. 
Although energy drink consumption is a significant public health concern in the UK, no UK based papers were located, a finding also noted in Visram et al's (2015) review. A review by Visram \& Hashem's (2016) draws on European and North American and Middle Eastern evidence, and makes several recommendations for UK practise, many focussed on school based interventions. School nurses could therefore make a substantial contribution to raising awareness of the risks associated with EDs, providing leadership in developing and implementing health promotion/awareness raising strategies

Studies that investigate the mechanism by which energy drinks counteract sleepiness in adolescents may provide further understanding of mechanisms of sleep regulation; investigation into both the short and long-term effects of using energy drinks as a counteractive measure for sleep on adolescents' health outcomes and academic performance are also recommended.

\section{Conclusion}

Despite current EU and UK regulatory measures on the labelling of energy drinks, consumption among UK adolescents continues to grow (ADEPIS, 2013, Visram \& Hashem, 2016). This review has reported on international literature examining behavioural correlates of ED consumption amongst adolescents to determine lessons that might be applied for practise in the UK. The cross-sectional nature of most of 
the studies limits findings to associations only; causality in the relationships discussed cannot be inferred. The studies mainly relied on young peoples' self-reports which, combined with recall bias, may influence accuracy of data, with over and underreporting of energy drink use, as well as misuse of alcohol and other substances possible. However, the studies included in the review drew on a range of young people drawn from different countries and communities, with most studies reporting findings based on sample sizes of more than 1000 participants.

The health risks associated with energy drinks are evident from this review, notably the risks around polysubstance use, alcohol dependence, binge drinking, alcohol-related aggressive behaviours, risky sexual behaviours, and alcohol-related accidents and injuries (O'Brien et al, 2008; Howland et al, 2011; Marczinski et al, 2011; Azagba et al, 2013; Howland and Rohsenow, 2013). Moreover, as the findings of the review suggest that energy drink consumption might be used as a risk-marker for alcohol and substance use among adolescents (Miyake and Marmorstein, 2014), frequent energy drink consumption may serve as a useful screening indicator to identify students at risk of substance use and/or other risky health behaviour (Miller, 2008). Enquiring about an adolescent's recent energy drink consumption could therefore be a non-confrontational means of commencing a discussion about alcohol use, which could facilitate opportunities for early intervention and prevention. 


\section{$\underline{\text { References }}$}

Alcohol and Drug Education and Prevention Information Service (ADEPIS) (2013) Drug and Alcohol Prevention: Caffeine and energy drinks. Available at: http://mentor-adepis.org/wpcontent/uploads/2013/09/Caffeine-energy-drinks.pdf Accessed: 29 June 2015

Arria, A., Bugbee, B., Caldeira, K. and Vincent, K. (2014) Evidence and knowledge gaps for the association between energy drink use and high-risk behaviors among adolescents and young adults. Nutrition Reviews, 72(1), 87-97.

Azagba, S., Langille, D. and Asbridge, M. (2013) The consumption of alcohol mixed with energy drinks: prevalence and key correlates among Canadian high school students. CMAJ Open, 1(1), 19-26.

Azagba, S., Langille, D. and Asbridge, M. (2014). An emerging adolescent health risk: caffeinated energy drink consumption patterns among high school students. Preventive Medicine, 6254-59

Beebe, D., Fallone, G., Godiwala, N., Flanigan, M., Martin, D., Schaffner, L. and Amin, R. (2008). Feasibility and behavioral effects of an at-home multi-night sleep restriction protocol for adolescents. Journal of Child Psychology \& Psychiatry, 49(9), 915-923.

Bonar, E. E., Cunningham, R. M., Polshkova, S., Chermack, S. T., Blow, F. C., \& Walton, M. A. (2015). Alcohol and energy drink use 
among adolescents seeking emergency department care. Addictive behaviors, 43, 11-17.

Brener, N. D., Billy, J. O., \& Grady, W. R. (2003). Assessment of factors affecting the validity of self-reported health-risk behavior among adolescents: evidence from the scientific literature. Journal of adolescent health, 33(6), 436-457.

Calamaro, C., Mason, T. and Ratcliffe, S. (2009) Adolescents living the 24/7 lifestyle: Effects of caffeine and technology on sleep duration and daytime functioning. Pediatrics, 123(6), 1005-1010.

Carrier, J., Paquet, J., Fernandez-Bolanos, M., Girouard, L., Roy, J., Selmaoui, B. and Filipini, D. (2009) Effects of caffeine on daytime recovery sleep: A double challenge to the sleep-wake cycle in aging. Sleep Medicine, 10(9), 1016-1024.

Crane, C. (2009) Energy Overload. Scholastic Choices, 24(4), 22-25.

Critical Appraisal Skills Programme (CASP) (2013). Critical Appraisal Skills Programme (CASP): Cohort Study Checklist. Available at: http://media.wix.com/ugd/dded87_e37a4ab637fe46a0869f9f977dacf1 $\underline{34 . p d f}$ 
(Assessed: 16 August 2015)

Dahl, R. and Lewin, D. (2002) Pathways to adolescent health: sleep regulation and behavior. Journal of Adolescent Health, 31(6), 175184.

Emond, J., Gilbert-Diamond., Tanski, S. and Sargent, J. (2014) Energy Drink Consumption and the Risk of Alcohol Use Disorder among a National Sample of Adolescents and Young Adults. Journal of Pediatrics, 165(6), 1194-1200.

Evren, C. and Evren, B. (2015) Energy-drink consumption and its relationship with substance use and sensation seeking among 10th grade students in Istanbul. Asian Journal of Psychiatry, 15, 44-50.

Food Standards Agency (2015) High caffeine energy drinks and other foods containing caffeine. Available at: https://www.food.gov.uk/science/additives/energydrinks (Accessed: 27 September 2015).

Gallimberti, L., Buja, A., Chindamo, S., Vinelli, A., Lazzarin, G., Terraneo, A., Scafato, E. and Baldo, V. (2013) Energy drink consumption in children and early adolescents. European Journal of Pediatrics, 172(10), 13-35. 
Hale, D.R. and Viner, R.M., 2012. Policy responses to multiple risk behaviours in adolescents. Journal of Public Health, 34(suppl 1), pp.i11-i19.

Hamilton, H., Boak, A., Ilie, G. and Mann, R. (2013) Energy drink consumption and associations with demographic characteristics, drug use and injury among adolescents. Canadian Journal of Public Health, 104(7). 496-501.

Hannes, K., Lockwood, C. and Pearson, A. (2010) A Comparative Analysis of Three Online Appraisal Instruments' Ability to Assess Validity in Qualitative Research. Qualitative Health Research, 20(12), 1736-1743.

Hawker, S., Payne, S., Kerr, C., Hardey, M. and Powell, J. (2002) Appraising the evidence: reviewing disparate data systematically. Qualitative Health Research, 12(9), 1284-1299.

Howland, J. and Rohsenow, D. J. (2013) Risks of energy drinks mixed with alcohol. JAMA, 309(3), 245-246.

Howland, J., Rohsenow, D., Calise, T., Mackillop, J. and Metrik, J. (2011) Caffeinated alcoholic beverages an emerging public health problem. American Journal of Preventive Medicine, 40(2), 268-271. Jackson, D., Cotter, B., Merchant, R., Babu, K., Baird, J., Nirenberg, T. and Linakis, J. (2013) Behavioral and physiologic adverse effects 
in adolescent and young adult emergency department patients reporting use of energy drinks and caffeine. Clinical Toxicology, $51(7), 557-565$

Larson, N., DeWolfe, J., Story, M. and Neumark-Sztainer, D. (2014) Adolescent Consumption of Sports and Energy Drinks: Linkages to Higher Physical Activity, Unhealthy Beverage Patterns, Cigarette Smoking, and Screen Media Use. Journal of Nutrition Education \& Behavior, 46(3), 181-187.

Leather, N. C. (2009). Risk-taking behaviour in adolescence: a literature review. Journal of Child Health Care, 13(3), 295-304.

Lodato, F., Araujo, J., Barros, H., Lopes, C., Agodi, A., Barchitta, M. and Ramos, E. (2013) Caffeine intake reduces sleep duration in adolescents. Nutrition Research, 33(9), 726-732.

Mann, M.J., Smith, M.L. and Kristjansson, A.L., 2016. Energy drink consumption and substance use risk in middle school students. Preventive Medicine Reports, 3, pp.279-282.

Marczinski, C., Fillmore, M., Bardgett, M. and Howard, M. (2011) Effects of energy drinks mixed with alcohol on behavioral control: Risks for college students consuming trendy cocktails. Alcoholism: Clinical and Experimental Research, 35(7), 1282-1292. 
Miller, K. (2008) Wired: energy drinks, jock identity, masculine norms, and risk taking. Journal of American College Health, 56(5), 481-489.

Miyake, E. and Marmorstein, N. (2015). Energy drink consumption and later alcohol use among early adolescents. Addictive Behaviors, 43(1), 60-65.

Nowak, D. and Jasionowski, A., (2015). Analysis of the consumption of caffeinated energy drinks among Polish adolescents. International journal of environmental research and public health, 12(7), pp.79107921.

O'Brien, M., McCoy, T., Rhodes, S., Wagoner, A. and Wolfson, M. (2008). Caffeinated Cocktails: Energy Drink Consumption, High-risk Drinking, and Alcohol-related consequences among College Students. Academic Emergency Medicine, 15(5), 453-460.

Orbeta, R., Overpeck, M., Ramcharran, D., Kogan, M. and Ledsky, R. (2006). High caffeine intake in adolescents: Associations with difficulty sleeping and feeling tired in the morning. Journal of Adolescent Health, 38(4), 451-453.

Patrick, M. and Maggs, J. (2014). Energy drinks and alcohol: Links to alcohol behaviors and consequences across 56 days. Journal of Adolescent Health, 54(4), 454-459. 
Pennington, N., Johnson, M., Delaney, E. and Blankenship, M. (2010). Energy drinks: A new health hazard for adolescents. The Journal of School Nursing, 26(5), 352-359.

Reissig, C., Strain, E. and Griffiths, R. (2009). Caffeinated energy drinks--a growing problem. Drug And Alcohol Dependence, 99(1), 110.

Rockett, I. and Putnam, S. (2002). Caffeine 'addiction' in high school youth: Evidence of an adverse health relationship. Addiction Research \& Theory, 10(1), 31-42.

Ruxton, C. (2014). The suitability of caffeinated drinks for children: a systematic review of randomised controlled trials, observational studies and expert panel guidelines. Journal of Human Nutrition \& Dietetics, 27(4), 342-357.

Schwartz, D., Gilstad-Hayden, K., Carroll-Scott, A., Grilo, S., McCaslin, C., Schwartz, M. and Ickovics, J. (2015). Energy Drinks and Youth Self-Reported Hyperactivity/Inattention Symptoms. Academic Pediatrics, 15(3), 297-304.

Seifert, S. M., Schaechter, J. L., Hershorin, E. R. and Lipschultz, S. (2011). Health Effects of Energy Drinks on Children, Adolescents, and Young Adults. Pediatrics, 127(3), 511-528

Snipes, D and Benotsch, E. (2013). High-risk cocktails and high-risk sex: examining the relation between alcohol mixed with energy drink 
consumption, sexual behavior, and drug use in college students. Addictive Behaviors, 38(1), 1418-1423.

Substance Abuse and Mental Health Service Administration. The DAWN Report: Update on Emergency Department Visits Involving Energy Drinks: A Continuing Public Health Concern. Rockville: 2013. Temple, J. (2009). Caffeine use in children: What we know, what we have left to learn, and why we should worry. Neuroscience and Biobehavioral Reviews, 33(6), 793-806.

Terry-McElrath, Y., O’Malley, P. and Johnston, L. (2014). Energy drinks, soft drinks, and substance use among United States secondary school students. Journal of Addiction Medicine, 8(1), 6-13.

Trapp, G., Allen, K., O'Sullivan, T., Robinson, M., Jacoby, P. and Oddy, W. (2014). Energy drink consumption among young Australian adults: associations with alcohol and illicit drug use. Drug And Alcohol Dependence, 134(1), 30-37.

Van Batenburg-Eddes, T., Lee, N., Weeda, W., Krabbendam, L., Huizinga, M., Mackiewicz Seghete, K. and Wilhelm, P. (2014). The potential adverse effect of energy drinks on executive functions in early adolescence. Frontiers in Psychology, 5(1), 1.

Van de Mortel, T. F. (2008). Faking it: social desirability response bias in self-report research. Australian Journal of Advanced Nursing, The, 25(4), 40 
Visram, S., Cheetham, M., Riby, D., Lake, A. A., \& Crossley, S. J.

(2015). A systematic review of the effects associated with children and young people's use of energy drinks. The European Journal of Public Health, 25(suppl 3), ckv173-070.

Visram, S., \& Hashem, K. (2016). Energy drinks: what's the evidence. Briefing Paper. London: Food Research Collaboration.

Wolk, B., Ganetsky, M. and Babu, K. (2012). Toxicity of energy drinks. Current Opinion in Pediatrics, 24(2), 24 